International Journal of Environment, Agriculture and Biotechnology
Vol-6, Issue-6; Nov-Dec, 2021
J Journal Home Page Available: $\underline{\text { https://ijeab.com/ }}$
Journal DOI: $10.22161 /$ ijeab

\title{
Effect of drinking saline water on performance, digestibility and nitrogen utilization of growing camels feed different quality roughages
}

\author{
A. M. Abdel-Wahed
}

Animal Nutrition Department, Animal \& Poultry Production Division, Desert Research Center, Cairo, Egypt

Received: 25 Sep 2021; Received in revised form: 07 Nov 2021; Accepted: 16 Nov 2021; Available online: 22 Nov 2021 C2021 The Author(s). Published by Infogain Publication. This is an open access article under the CC BY license (https://creativecommons.org/licenses/by/4.0/).

\begin{abstract}
This study was conducted to evaluate the effect of three roughages that were fed ad lib. with two types of water on feed intake, digestibility, water utilization and performance of growing she-camels. Twenty-four healthy growing she-camels $(30-36$ months old and $448.50 \pm 29.30 \mathrm{~kg}$ body weight) were housed individually in metabolic cages and randomly allotted to three treatments. The experiment lasted for 60 days. Three roughages were Egyptian clover hay to represent optimum grazing conditions, rice straw to represent dry season grazing and Atriplex halimus to represent arid rangelands dominated by halophytes. Roughages offered to camels ad lib. The concentrates used were corn grains and cottonseed meal. Concentrate intakes calculated, per unit metabolic body weight (kg0.73). Final body weight and ADG were affected by roughages. Nutrients intake was affected $(P<0.05)$ by roughage type but not for drinking water and their interaction. Camels fed Atriplex had higher $(P<0.05)$ in dry matter intake, roughage intake and roughage $(\%$ DMI) than hay and straw. Corn intake was greater $(P<0.05)$ in camels fed Atriplex and straw whereas, was lower $(P<0.05)$ in camels fed hay. Camels fed hay had higher $(P<0.05)$ in dry matter digestibility followed by straw and then Atriplex. Organic matter, crude fiber and Nitrogen free extract digestibility were higher $(P<0.05)$ in camels fed hay and straw as compared to Atriplex whereas, crude protein was higher $(P<0.05)$ in Atriplex and hay as compared to straw. Free water intake, feed water, total water intake and fecal water were higher $(P<0.05)$ in camels fed Atriplex as compared to hay and straw. Urinary water was higher $(P<0.05)$ in camels fed Atriplex followed by hay and then straw. Free water intake, feed water, total water intake and fecal water were higher $(P<0.05)$ in camels fed Atriplex as compared to hay and straw. Urinary water excretion was higher $(P<0.05)$ in camels fed Atriplex followed by hay and then straw. In conclusion, camels fed on Atriplex showed a clear improvement in growth, digestibility, and nitrogen utilization in a similar way to camels fed on hay.
\end{abstract}

Keywords - growing she-camels, roughages type, water type, intake, performance, water utilization.

\section{INTRODUCTION}

Dromedary camel (Camelus dromedarius) is an important livestock species that is exceptionally well adapted to harsh environmental conditions. They are functionally and metabolically ruminant herbivores. Ruminant herbivores are distinguished by their multipart stomach. This anatomical structure and the microorganisms that inhabit the rumen network allow for longer retention of ingested feed and anaerobic microbial digestion of cellulosic materials, and consequently production of volatile fatty acids and microbial protein synthesis. Camels are bred because of their extraordinary strength to withstand hunger and thirst for a long time in the environmental conditions [1] In addition, its high ability to convert scarce desert resources into milk and meat makes it even more important to pastoralists [2,3].

Camels are non-selective grazers with a digestive system that has evolved - adapt to unfavorable conditions and has a greater activity of cellulolytic bacteria [6,7] Camels had a greater capacity to utilize low-quality roughages that are high in NDF and ADF and less digestible[6,7]. Camels are typically associated with a 
lower feed intake and greater efficiency of forage utilization, which may be due to their large body size and longer retention time, which gives more opportunity for microorganisms to digest non-structure carbohydrates [7]. On the other hand, camels prefer to consume salty bushes which are rich in moisture and salt. Salts present in such plants help to meet the physiological functions of camels $[8,9]$ reported that the high moisture content of salt bushes ensures a good portion of the camel water requirement in areas where water is the most limiting factor for animals. Feeding halophytes especially for camels can be an appropriate method in arid regions to reduce the problem of forage shortage. Camels have adapted rumen microbial communities that enable them to take advantage of the non-protein nitrogen found in halophytes.

Camels have adapted mechanisms that allow it to withstand prolonged water deprivation especially in the absence of readily available water and survive when feed resources are scarce or of poor quality [10]. Camels are able to replenish in a relative short period of time the water lost. Whereas changes of water metabolism, body fluid and its regulation, body temperature, kidney function, appetite and hormonal aspects during dehydration have been studied in the past [11-14].

Lack of forage and water deprivation are important barriers to camel production in arid and semiarid regions of the harsh climate. However, they are slowly being replaced by stable systems which should properly take into account the feeding of camels in these systems. Therefore, the present study was conducted to evaluate the effect of different forages and types of water on intake, nutrient digestibility, and performance of camels.

\section{MATERIALS AND METHODS}

The experiment was conducted at Maryout Research Station, Desert Research Center, Alexandria, Egypt.

Table 1. Proximate composition of feed ingredients, \% DM basis.

\begin{tabular}{lccccc}
\hline \multicolumn{1}{c}{$\begin{array}{c}\text { Proximate } \\
\text { Constituents }\end{array}$} & $\begin{array}{c}\text { Corn } \\
\text { grains }\end{array}$ & $\begin{array}{c}\text { Cottonseed } \\
\text { meal }^{\mathbf{1}}\end{array}$ & $\begin{array}{c}\text { Egyptian } \\
\text { clover hay }\end{array}$ & $\begin{array}{c}\text { Atriplex } \\
\text { halimus }^{2}\end{array}$ & $\begin{array}{c}\text { Rice } \\
\text { straw }\end{array}$ \\
\hline Dry matter & 86.65 & 90.88 & 86.08 & 34.98 & 87.43 \\
Ash & 1.71 & 24.73 & 13.35 & 25.37 & 21.68 \\
Organic matter & 98.29 & 75.27 & 86.65 & 74.99 & 78.32 \\
Crude protein & 10.76 & 15.84 & 14.26 & 11.70 & 4.55 \\
Crude fiber & 3.77 & 19.30 & 34.23 & 28.62 & 28.86 \\
Ether extract & 3.92 & 10.86 & 4.40 & 2.94 & 2.52 \\
N-free extract & 79.84 & 29.27 & 33.76 & 31.37 & 42.39 \\
\hline${ }^{1}$ Un-decorticated, heat treated and mechanically pressed CSM, produced in a traditional oil mill, \\
${ }^{2}$ Leaves and succulent branches typically consumed by grazing animals.
\end{tabular}

\subsection{Digestion trials}

At the end of the experimental period, camels were placed in metabolic cages for 15 days, 8 days of adaptation to the

\subsection{Animals, diets, and experimental design}

Twenty-four healthy growing she-camels (Camelus dromedarius) with an average initial body weight (BW) of $448.50 \pm 29.30 \mathrm{~kg}$ and 30-36 months old were used and the experiment lasted for 60 days. Animals were housed individually in shaded floor pens for the duration of the experiment. The experiment was arranged as a $3 \times 2$ factorial experiment in a completely randomized design by using three forages and two types of water. Animal were weighed every two weeks after overnight fast and on two consecutive days and the average daily gain (ADG) was calculated.

The three roughages were used to represent the prevailing different grazing conditions in arid rangelands. Those were Egyptian clover hay to represent optimum grazing conditions, rice straw to represent dry season grazing and Atriplex halimus to represent arid rangelands dominated by halophytes. The concentrates used were corn grains and cottonseed meal selected as the commonly used energy and protein supplements, respectively. Roughages offered to camels ad lib. twice daily at 8:00 and 16:00 hours. Refusals were weighed at the following morning and daily intake was recorded on dry matter basis. Concentrate

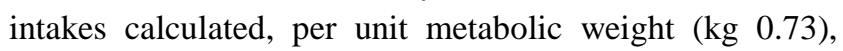
from a previous experiment [15], actual intake is presented below. in an attempt to control anticipated excessive soluble carbohydrates intake and possible adverse effects on rumen function and feed utilization [15]. The animals are drunk once every day, either tap water or salty 10,000 parts per million. The proximate composition of feed ingredients is presented in Table 1. Refusals were weighed daily, and feed intake was recorded. Samples of the roughages and concentrates were collected and analyzed for DM by drying to constant weight in a forced-air oven at $60^{\circ} \mathrm{C}$ for $48 \mathrm{~h}$ [16]. Samples were pooled for each camel. 
faecal output was analyzed for DM by drying to constant weight in a forced-air oven at $60^{\circ} \mathrm{C}$ for $48 \mathrm{~h}$ [16]. Dried ingredients, orts and faecal samples were ground in a Wiley mill with a 1-mm screen. Samples were analyzed for ash, ether extract, crude fiber and crude protein according to [16]. Urine samples were collected in plastic containers containing $100 \mathrm{ml}$ of $\mathrm{H} 2 \mathrm{SO} 4$. Total daily urine output was weighed and recorded. A $10 \%$ subsample was collected and then analyzed for $\mathrm{N}$ [16].

\subsection{Statistical analysis}

Main effects and interactions were evaluated using the GLM repeated-measures analysis of variance procedures of the NCSS statistical package [17]. The type of roughage and concentrate levels were the independent variables, and type of water (water tap and saline water) levels were repeated within roughages. Newman-Keuls multiple comparison tests was applied to the means of the main effects, i.e. type of roughage, R-means, and level of concentrates, type of water (water tap and saline water) Bmeans. Statistical significance was declared at $\mathrm{P} \leq 0.05$.

\section{RESULTS}

Chemical composition of the roughages is presented in Table 1. Three forages were selected differ in their crude protein content. Crude protein was higher for clover hay, intermediate for the Atriplex halimus, and lowest for rice straw. Two concentrates were selected differ in their chemical composition Table 1. Cottonseed meal was higher in their contents of crude protein, ash, crude fiber, and lower in nitrogen free extract compared with corn grain.

Effects of roughages type and drinking saline water on the performance of the camels are presented in Table 2. Initial body weight was not affected whereas, final body weight and ADG were affected by roughages type $(\mathrm{P}<0.05)$ but not for drinking water and their interaction.

Effects of roughages type and drinking saline water on the feed intake of the camels are presented in Table 3. Feed intake was affected $(\mathrm{P}<0.05)$ by roughage type but not for drinking water and their interaction. Camels fed Atriplex had higher $(\mathrm{P}<0.05)$ intake of dry matter, roughage intake and roughage $(\% \mathrm{DMI})$ than hay and straw. Corn intake was greater $(\mathrm{P}<0.05)$ in camels fed Atriplex and straw whereas, was lower $(\mathrm{P}<0.05)$ in camels fed hay.

Effects of roughages type and drinking saline water on the components of are presented in Table 4. Organic matter, crude protein, crude fiber, rumen degradable protein, rumen un-degradable protein intakes were affected $(\mathrm{P}<0.05)$ by roughage type but not for drinking water and their interaction. Camels fed Atriplex had higher values $(\mathrm{P}<0.05)$ of organic matter intake and crude fiber intake than hay and straw. Crude protein intake, rumen degradable protein intake and rumen un-degradable protein were greater $(\mathrm{P}<0.05)$ in camels fed Atriplex and hay whereas, were lower $(\mathrm{P}<0.05)$ in camels fed straw.

Effects of roughages type and drinking saline water on the apparent digestion coefficients of diets consumed by camels are presented in Table 5. Digestion coefficients of diets was affected $(\mathrm{P}<0.05)$ by roughage type but not for drinking water and their interaction. Camels fed hay had higher $(\mathrm{P}<0.05)$ in dry matter digestibility followed by straw and then Atriplex. Organic matter, crude fiber and nitrogen free extract digestibility were higher $(\mathrm{P}<0.05)$ in camels fed hay and straw as compared to Atriplex whereas, crude protein was higher $(\mathrm{P}<0.05)$ in Atriplex and hay as compared to straw.

Effects of roughages type and drinking saline water on the nitrogen utilization are presented in Table 6. Nitrogen utilization was affected $(\mathrm{P}<0.05)$ by roughage type but not for drinking water and their interaction. Nitrogen intake and digested nitrogen were higher $(\mathrm{P}<0.05)$ in camels fed hay and Atriplex as compared to straw whereas, fecal nitrogen, urinary nitrogen and nitrogen balance were not affected.

Effects of roughages type and drinking saline water on the water intake and execration are presented in Tables 7 and 8 Water intake and execration were affected $(\mathrm{P}<0.05)$ by roughage type but not for drinking water and their interaction. Free water intake, feed water, total water intake and fecal water were higher $(\mathrm{P}<0.05)$ in camels fed Atriplex as compared to hay and straw. Urinary water was higher $(\mathrm{P}<0.05)$ in camels fed Atriplex followed by hay and then straw.

\section{DISCUSSIONS}

The present experiment showed that final body weight and ADG were affected by the type of roughages. Similar results were observed by [18] who reported that ADG g/day of camels fed hay and Atriplex were higher than those of their mates fed rice straw with limiting concentrate offered to $50 \%$. In agreement with $[19,15,7]$ the straw is characterized by its poor of digestion, a longer retention time in the rumen and its low nutritional value, which negatively affected the performance.

Effect of saline water on ADG camels fed straw and drinking salt water was lower final body weight and ADG Similar findings were reported by [20] who reported that female camels lost $1.9 \%$ of their initial live body weight when drinking saline water. 
Our results indicate that camels consumed higher amounts of Atriplex compared to hay and straw. This is consistent with similar findings found by [21]. [22] reported that camels need salt more than other livestock in their diets, which they get from Atriplex, which contain salts that may reach $25 \%$ of DM [23].

Table 2. Effect of type roughages and drinking water and saline water on the performance of the camels.

\begin{tabular}{|c|c|c|c|c|c|c|c|}
\hline \multirow{2}{*}{ Water type (B) } & \multicolumn{3}{|c|}{ Roughage, ad lib. (R) } & \multirow{2}{*}{$\begin{array}{c}\text { Water } \\
\text { Average }\end{array}$} & \multicolumn{3}{|c|}{$P$-value ${ }^{1}$} \\
\hline & Atriplex & hay & Straw & & $\mathbf{R}$ & B & $\mathbf{R x B}$ \\
\hline \multicolumn{8}{|l|}{ Initial body weight (kg) } \\
\hline Fresh & 448 & 446 & 446 & 447 & 0.35 & 0.61 & 0.33 \\
\hline Saline & 446 & 447 & 446 & 446 & & & \\
\hline Roughage average & 447 & 446 & 446 & 447 & & & \\
\hline \pm SEM & 15.9 & & & & & & \\
\hline \multicolumn{8}{|l|}{ Final body weight (kg) } \\
\hline Fresh & 480 & 483 & 468 & 477 & $<0.01$ & 0.43 & 0.21 \\
\hline Saline & 470 & 480 & 456 & 469 & & & \\
\hline Roughage average & $475^{\mathrm{a}}$ & $481^{\mathrm{a}}$ & $462^{\mathrm{b}}$ & 473 & & & \\
\hline \pm SEM & 85.8 & & & & & & \\
\hline \multicolumn{8}{|l|}{ Average daily gain (g/day) } \\
\hline Fresh & 531 & 608 & 360 & 500 & $<0.01$ & 0.52 & 0.13 \\
\hline Saline & 400 & 550 & 172 & 374 & & & \\
\hline Roughage average & $465^{\mathrm{a}}$ & $579^{\mathrm{a}}$ & $266^{\mathrm{b}}$ & 437 & & & \\
\hline \pm SEM & 74.5 & & & & & & \\
\hline
\end{tabular}

Table 3. Average daily feed intake during the digestion trials, $\mathrm{g} / \mathrm{d} / \mathrm{kg}^{0.75}$

\begin{tabular}{|c|c|c|c|c|c|c|c|}
\hline \multirow{2}{*}{ Water type (B) } & \multicolumn{3}{|c|}{ Roughage, ad lib. (R) } & \multirow{2}{*}{$\begin{array}{c}\text { Water } \\
\text { average }\end{array}$} & \multicolumn{3}{|c|}{$P$-value ${ }^{1}$} \\
\hline & Atriplex & Hay & Straw & & $\mathbf{R}$ & B & $\mathbf{R x B}$ \\
\hline \multicolumn{8}{|l|}{ Dry matter intake(DMI) } \\
\hline Fresh & 85.5 & 63.5 & 56.0 & 68.3 & $<0.01$ & 0.29 & 0.45 \\
\hline Saline & 96.9 & 63.4 & 57.9 & 72.7 & & & \\
\hline Roughage average & $91.2^{\mathrm{a}}$ & $63.4^{\mathrm{b}}$ & $56.9^{\mathrm{b}}$ & 70.5 & & & \\
\hline \pm SEM & 4.58 & & & & & & \\
\hline \multicolumn{8}{|l|}{ Roughage (DMI) } \\
\hline Fresh & 56.3 & 34.7 & 27.1 & 39.4 & $<0.01$ & 0.28 & 0.38 \\
\hline Saline & 67.6 & 34.6 & 28.1 & 43.4 & & & \\
\hline Roughage average & $61.9^{\mathrm{a}}$ & $34.6^{\mathrm{b}}$ & $27.6^{\mathrm{b}}$ & 41.4 & & & \\
\hline \pm SEM & 4.18 & & & & & & \\
\hline \multicolumn{8}{|l|}{ Roughage (\% in DMI) } \\
\hline Fresh & 65.5 & 54.6 & 48.3 & 56.1 & $<0.01$ & 0.28 & 0.34 \\
\hline Saline & 69.8 & 54.6 & 48.5 & 57.6 & & & \\
\hline Roughage average & $67.6^{\mathrm{a}}$ & $54.6^{\mathrm{b}}$ & $48.4^{\mathrm{c}}$ & 58.9 & & & \\
\hline \pm SEM & 1.51 & & & & & & \\
\hline \multicolumn{8}{|l|}{ Corn (DMI) } \\
\hline Fresh & 23.8 & 20.6 & 22.3 & 22.2 & 0.01 & 0.67 & 0.85 \\
\hline Saline & 23.9 & 20.6 & 22.9 & 22.5 & & & \\
\hline Roughage average & $23.8^{\mathrm{a}}$ & $20.6^{\mathrm{b}}$ & $22.6^{\mathrm{a}}$ & 22.3 & & & \\
\hline \pm SEM & 0.64 & & & & & & \\
\hline \multicolumn{8}{|l|}{ Cotton seed meal (DMI) } \\
\hline Fresh & 5.37 & 8.24 & 6.70 & 6.77 & $<0.01$ & 0.64 & 0.78 \\
\hline Saline & 5.39 & 8.22 & 6.90 & 6.84 & & & \\
\hline Roughage average & $5.38^{\mathrm{c}}$ & $8.23^{\mathrm{a}}$ & $6.80^{\mathrm{b}}$ & 6.80 & & & \\
\hline \pm SEM & 0.16 & & & & & & \\
\hline \multicolumn{8}{|l|}{ Concentrate (DMI) } \\
\hline Fresh & 29.2 & 28.8 & 28.9 & 28.9 & 0.67 & 0.66 & $0 . .83$ \\
\hline Saline & 29.3 & 28.8 & 29.8 & 29.3 & & & \\
\hline Roughage average & 29.2 & 28.8 & 29.4 & 29.1 & & & \\
\hline \pm SEM & 0.79 & & & & & & \\
\hline
\end{tabular}


Table 4. Components of diets $\mathrm{g} / \mathrm{d} / \mathrm{kg}^{0.73}$

\begin{tabular}{|c|c|c|c|c|c|c|c|}
\hline \multirow{2}{*}{ Water type (B) } & \multicolumn{3}{|c|}{ Roughage, ad lib. (R) } & \multirow{2}{*}{$\begin{array}{c}\text { Water } \\
\text { average }\end{array}$} & \multicolumn{3}{|c|}{$P$-value ${ }^{1}$} \\
\hline & Atriplex & Hay & Straw & & $\mathbf{R}$ & B & $\mathbf{R x} \mathbf{B}$ \\
\hline \multicolumn{8}{|c|}{ Organic matter intake (OMI) } \\
\hline Fresh & 69.5 & 56.6 & 49.6 & 58.6 & $<0.01$ & 0.29 & 0.47 \\
\hline Saline & 77.9 & 56.5 & 51.2 & 61.9 & & & \\
\hline Roughage average & $73.7^{\mathrm{a}}$ & $56.6^{\mathrm{b}}$ & $50.4^{\mathrm{b}}$ & 60.2 & & & \\
\hline \pm SEM & 3.51 & & & & & & \\
\hline \multicolumn{8}{|c|}{ Crude protein intake (CPI) } \\
\hline Fresh & 7.61 & 8.64 & 4.75 & 6.67 & $<0.01$ & 0.29 & 0.46 \\
\hline Saline & 8.47 & 8.61 & 4.89 & 7.33 & & & \\
\hline Roughage average & $8.04^{\mathrm{a}}$ & $8.62^{\mathrm{a}}$ & $4.82^{\mathrm{b}}$ & 7.16 & & & \\
\hline \pm SEM & 0.35 & & & & & & \\
\hline \multicolumn{8}{|l|}{ Crude fiber intake (CFI) } \\
\hline Fresh & 17.9 & 13.8 & 10.3 & 14.0 & $<0.01$ & 0.47 & 0.40 \\
\hline Saline & 21.1 & 13.8 & 10.6 & 15.2 & & & \\
\hline Roughage average & $19.5^{\mathrm{a}}$ & $13.8^{\mathrm{b}}$ & $10.4^{\mathrm{b}}$ & 14.6 & & & \\
\hline \pm SEM & 1.20 & & & & & & \\
\hline \multicolumn{8}{|c|}{ Rumen degradable protein intake (RDPI) } \\
\hline Fresh & 5.49 & 6.23 & 2.90 & 4.87 & $<0.01$ & 0.30 & 0.44 \\
\hline Saline & 6.13 & 6.21 & 2.99 & 5.11 & & & \\
\hline Roughage average & $5.81^{\mathrm{a}}$ & $6.22^{\mathrm{a}}$ & $2.94^{\mathrm{b}}$ & 4.99 & & & \\
\hline \pm SEM & 0.001 & & & & & & \\
\hline \multicolumn{8}{|c|}{ Rumen un-degradable protein (RUPI) } \\
\hline Fresh & 2.12 & 2.41 & 1.85 & 2.83 & 0.01 & 0.28 & 0.51 \\
\hline Saline & 2.34 & 2.40 & 1.91 & 2.22 & & & \\
\hline Roughage average & $2.23^{\mathrm{a}}$ & $2.40^{\mathrm{a}}$ & $1.88^{\mathrm{b}}$ & 2.17 & & & \\
\hline \pm SEM & 0.01 & & & & & & \\
\hline
\end{tabular}

Table 5. Apparent digestion coefficients of diets consumed by camels, \%

\begin{tabular}{|c|c|c|c|c|c|c|c|}
\hline \multirow{2}{*}{ Water type (B) } & \multicolumn{3}{|c|}{ Roughage, ad lib. (R) } & \multirow{2}{*}{$\begin{array}{c}\text { Water } \\
\text { average }\end{array}$} & \multicolumn{3}{|c|}{$P$-value ${ }^{1}$} \\
\hline & Atriplex & Hay & Straw & & $\mathbf{R}$ & B & $\mathbf{R x B}$ \\
\hline \multicolumn{8}{|l|}{ Dry matter } \\
\hline Fresh & 58.2 & 67.9 & 63.0 & 63.1 & 0.02 & 0.82 & 0.73 \\
\hline Saline & 58.1 & 71.2 & 61.6 & 63.6 & & & \\
\hline Roughage average & $58.1^{\mathrm{c}}$ & $69.6^{\mathrm{a}}$ & $62.3^{\mathrm{b}}$ & 63.3 & & & \\
\hline \pm SEM & 2.97 & & & & & & \\
\hline \multicolumn{8}{|l|}{ Organic matter } \\
\hline Fresh & 55.5 & 69.4 & 67.2 & 64.1 & 0.01 & 0.71 & 0.76 \\
\hline Saline & 54.6 & 72.8 & 67.5 & 64.9 & & & \\
\hline Roughage average & $55.1^{\mathrm{b}}$ & $71.1^{\mathrm{a}}$ & $67.3^{\mathrm{a}}$ & 64.5 & & & \\
\hline \pm SEM & 2.94 & & & & & & \\
\hline \multicolumn{8}{|l|}{ Crude protein } \\
\hline Fresh & 63.9 & 63.3 & 45.3 & 57.5 & 0.03 & 0.79 & 0.74 \\
\hline Saline & 60.4 & 65.9 & 49.7 & 58.7 & & & \\
\hline Roughage average & $62.1^{\mathrm{a}}$ & $64.6^{\mathrm{a}}$ & $47.5^{\mathrm{b}}$ & 58.1 & & & \\
\hline \pm SEM & 5.22 & & & & & & \\
\hline \multicolumn{8}{|l|}{ Crude fiber } \\
\hline Fresh & 28.8 & 57.5 & 58.4 & 48.2 & 0.01 & 0.42 & 0.51 \\
\hline Saline & 37.6 & 66.1 & 54.1 & 52.6 & & & \\
\hline Roughage average & $33.2^{\mathrm{b}}$ & $61.8^{\mathrm{a}}$ & $56.3^{\mathrm{a}}$ & 50.4 & & & \\
\hline \pm SEM & 6.10 & & & & & & \\
\hline \multicolumn{8}{|l|}{ Nitrogen free extract } \\
\hline Fresh & 63.5 & 75.5 & 72.6 & 70.5 & 0.03 & 0.71 & 0.59 \\
\hline Saline & 59.6 & 76.6 & 73.1 & 69.8 & & & \\
\hline Roughage average & $61.6^{\mathrm{b}}$ & $76.1^{\mathrm{a}}$ & $72.8^{\mathrm{a}}$ & 70.2 & & & \\
\hline \pm SEM & 2.48 & & & & & & \\
\hline
\end{tabular}


Table 6. Nitrogen utilization, $\mathrm{mg}^{\mathrm{N}} / \mathrm{day} / \mathrm{kg}^{0.73}$

\begin{tabular}{|c|c|c|c|c|c|c|c|}
\hline \multirow{2}{*}{ Water type (B) } & \multicolumn{3}{|c|}{ Roughage, ad lib. (R) } & \multirow{2}{*}{$\begin{array}{c}\text { Water } \\
\text { average }\end{array}$} & \multicolumn{3}{|c|}{$P$-value ${ }^{1}$} \\
\hline & Atriplex & Hay & Straw & & $\mathbf{R}$ & B & $\mathbf{R x B}$ \\
\hline \multicolumn{8}{|l|}{ Nitrogen intake } \\
\hline Fresh & 1217 & 1382 & 760 & 1120 & $<0.01$ & 0.29 & 0.46 \\
\hline Saline & 1355 & 1378 & 783 & 1172 & & & \\
\hline Roughage average & $1286^{\mathrm{a}}$ & $1380^{\mathrm{a}}$ & $772^{\mathrm{b}}$ & 1145 & & & \\
\hline \pm SEM & 56.1 & & & & & & \\
\hline \multicolumn{8}{|l|}{ Fecal nitrogen } \\
\hline Fresh & 432 & 507 & 415 & 451 & 0.16 & 0.66 & 0.26 \\
\hline Saline & 538 & 470 & 393 & 467 & & & \\
\hline Roughage average & 485 & 488 & 404 & 459 & & & \\
\hline \pm SEM & 42.4 & & & & & & \\
\hline \multicolumn{8}{|l|}{ Digested nitrogen } \\
\hline Fresh & 785 & 875 & 345 & 668 & $<0.01$ & 0.59 & 0.99 \\
\hline Saline & 817 & 908 & 390 & 705 & & & \\
\hline Roughage average & $801^{\mathrm{a}}$ & $891^{\mathrm{a}}$ & $368^{\mathrm{b}}$ & 687 & & & \\
\hline \pm SEM & 78.6 & & & & & & \\
\hline \multicolumn{8}{|l|}{ Urinary nitrogen } \\
\hline Fresh & 457 & 734 & 240 & 477 & 0.11 & 0.74 & 0.34 \\
\hline Saline & 763 & 521 & 285 & 523 & & & \\
\hline Roughage average & 610 & 627 & 263 & 500 & & & \\
\hline \pm SEM & 159 & & & & & & \\
\hline \multicolumn{8}{|l|}{ Nitrogen balance } \\
\hline Fresh & 328 & 141 & 105 & 191 & 0.66 & 0.95 & 0.38 \\
\hline Saline & 54.6 & 387 & 105 & 183 & & & \\
\hline Roughage average & 191 & 264 & 105 & 187 & & & \\
\hline \pm SEM & 172 & & & & & & \\
\hline
\end{tabular}

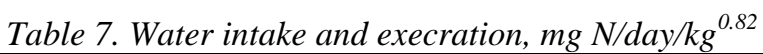

\begin{tabular}{|c|c|c|c|c|c|c|c|}
\hline \multirow{2}{*}{ Water type (B) } & \multicolumn{3}{|c|}{ Roughage, ad lib. (R) } & \multirow{2}{*}{$\begin{array}{c}\text { Water } \\
\text { average }\end{array}$} & \multicolumn{3}{|c|}{$P$-value ${ }^{1}$} \\
\hline & Atriplex & Hay & Straw & & $\mathbf{R}$ & B & $\mathbf{R x} B$ \\
\hline \multicolumn{8}{|l|}{ Free water intake } \\
\hline Fresh & 148 & 111 & 125 & 128 & 0.01 & 0.23 & 0.63 \\
\hline Saline & 143 & 108 & 108 & 119 & & & \\
\hline Roughage average & $146^{\mathrm{a}}$ & $109^{\mathrm{b}}$ & $116^{\mathrm{b}}$ & 124 & & & \\
\hline \pm SEM & 7.69 & & & & & & \\
\hline \multicolumn{8}{|l|}{ Feed water } \\
\hline Fresh & 73.5 & 20.2 & 13.5 & 35.7 & $<0.01$ & 0.87 & 0.58 \\
\hline Saline & 75.3 & 16.8 & 14.1 & 35.4 & & & \\
\hline Roughage average & $74.4^{\mathrm{a}}$ & $18.5^{\mathrm{b}}$ & $13.8^{\mathrm{b}}$ & 35.6 & & & \\
\hline \pm SEM & 2.48 & & & & & & \\
\hline \multicolumn{8}{|l|}{ Total water intake } \\
\hline Fresh & 234 & 144 & 149 & 176 & $<0.01$ & 0.34 & 0.73 \\
\hline Saline & 233 & 138 & 133 & 168 & & & \\
\hline Roughage average & $233^{\mathrm{a}}$ & $141^{\mathrm{b}}$ & $141^{\mathrm{b}}$ & 172 & & & \\
\hline \pm SEM & 9.21 & & & & & & \\
\hline \multicolumn{8}{|l|}{ Fecal water } \\
\hline Fresh & 29.8 & 17.4 & 12.7 & 19.9 & $<0.01$ & 0.46 & 0.27 \\
\hline Saline & 36.6 & 13.5 & 15.6 & 21.9 & & & \\
\hline Roughage average & $33.2^{\mathrm{a}}$ & $15.4^{\mathrm{b}}$ & $14.1^{\mathrm{b}}$ & 20.9 & & & \\
\hline \pm SEM & 3.02 & & & & & & \\
\hline \multicolumn{8}{|l|}{ Urinary water } \\
\hline Fresh & 45.1 & 56.9 & 29.3 & 43.8 & 0.02 & 0.11 & 0.04 \\
\hline Saline & 110 & 44.7 & 32.4 & 62.4 & & & \\
\hline Roughage average & $77.6^{\mathrm{a}}$ & $50.8^{\mathrm{b}}$ & $30.8^{\mathrm{c}}$ & 53.1 & & & \\
\hline \pm SEM & 11.9 & & & & & & \\
\hline
\end{tabular}


Table 8. water intake, $\mathrm{ml} / \mathrm{day} / \mathrm{kg}^{0.82}$

\begin{tabular}{|c|c|c|c|c|c|c|c|}
\hline \multirow{2}{*}{ Water type (B) } & \multicolumn{3}{|c|}{ Roughage, ad lib. (R) } & \multirow{2}{*}{$\begin{array}{c}\text { Water } \\
\text { average }\end{array}$} & \multicolumn{3}{|c|}{$P$-value ${ }^{1}$} \\
\hline & Atriplex & hay & Straw & & $\mathbf{R}$ & B & $\mathbf{R x} \mathbf{B}$ \\
\hline \multicolumn{8}{|c|}{ Free water intake (FWI) } \\
\hline Fresh & 147.74 & 110.97 & 124.74 & 127.82 & 0.007 & 0.233 & 0.628 \\
\hline Saline & 143.32 & 107.65 & 107.55 & 119.51 & & & \\
\hline Roughage average & $145.53^{\mathrm{a}}$ & $109.31^{\mathrm{b}}$ & $116.14^{\mathrm{b}}$ & 123.66 & & & \\
\hline \pm SEM & 7.687 & & & & & & \\
\hline \multicolumn{8}{|l|}{ Feed water (FDWI) } \\
\hline Fresh & 73.48 & 20.18 & 13.54 & 35.73 & 0.0000 & 0.875 & 0.577 \\
\hline Saline & 75.32 & 16.78 & 14.11 & 35.40 & & & \\
\hline Roughage average & $74.40^{\mathrm{a}}$ & $18.48^{\mathrm{b}}$ & $13.82^{\mathrm{b}}$ & 35.57 & & & \\
\hline \pm SEM & 2.482 & & & & & & \\
\hline \multicolumn{8}{|c|}{ Metabolic water (MWI) } \\
\hline Fresh & 12.73 & 12.95 & 11.02 & 12.23 & 0.254 & 0.457 & 0.937 \\
\hline Saline & 14.07 & 13.50 & 11.57 & 13.05 & & & \\
\hline Roughage average & 13.40 & 13.22 & 11.29 & 12.64 & & & \\
\hline \pm SEM & 1.255 & & & & & & \\
\hline \multicolumn{8}{|c|}{ Total water intake (TWI) } \\
\hline Fresh & 233.96 & 144.10 & 149.30 & 175.79 & 0.0007 & 0.339 & 0.727 \\
\hline Saline & 232.71 & 137.93 & 133.23 & 167.96 & & & \\
\hline Roughage average & $233.34^{\mathrm{a}}$ & $141.02^{\mathrm{b}}$ & $141.27^{\mathrm{b}}$ & 171.87 & & & \\
\hline \pm SEM & 9.208 & & & & & & \\
\hline \multicolumn{8}{|l|}{ TWI/GE } \\
\hline Fresh & 1.256 & 0.915 & 1.112 & $1.096^{\mathrm{a}}$ & 0.001 & 0.013 & 0.272 \\
\hline Saline & 1.108 & 0.885 & 0.961 & $0.984^{\mathrm{b}}$ & & & \\
\hline Roughage average & $1.182^{\mathrm{a}}$ & $0.899^{\mathrm{c}}$ & $1.039^{\mathrm{b}}$ & 1.040 & & & \\
\hline \pm SEM & 0.0392 & & & & & & \\
\hline \multicolumn{8}{|l|}{ TWI/DE } \\
\hline Fresh & 2.229 & 1.313 & 1.669 & 1.737 & 0.002 & 0.130 & 0.836 \\
\hline Saline & 1.991 & 1.212 & 1.415 & 1.539 & & & \\
\hline Roughage average & $2.110^{\mathrm{a}}$ & $1.262^{\mathrm{b}}$ & $1.542^{\mathrm{b}}$ & 1.638 & & & \\
\hline \pm SEM & 0.1381 & & & & & & \\
\hline
\end{tabular}

This was confirmed by [24] who explained that camels need 6 to 8 times the amount of salt than other livestock requirement, and also indicated that camels that do not regularly receive salty feed need about 140 grams of salt per dat. Moreover, Atriplex is a green plant more palatable and preferred for camels than alfalfa hay and rice straw as indicated by [22].

Our results indicate that the camels fed on clover hay had higher digestibility values, as clover hay is considered a good-quality roughage that is high in its available content of nutrients, which explains the high digestion parameters. These results are consistent with [18]

Camels can efficiently digest low quality roughage that is low in nutritional value, which explains the high fiber digestibility coefficient of camels fed straw.The more efficient utilization of low quality roughage by camels is mainly the result of a higher cellulolytic activity of the microorganisms [5] and to a longer retention time of solid particles [6,7]. Camels fed Atriplex showed improved protein digestibility, which may be due to their content of non-protein nitrogen (NPN). [7] reported that Atriplex differs from conventional forage in terms of its high NPN content and About $44.5 \%$ of the protein content is NPN. The NPN compounds are degraded and used as a source of $\mathrm{N}$ for the synthesis of microbial protein. The effective uptake of non-protein nitrogen by rumen microbes and subsequent conversion to microbial protein, diets should contain suitable and adequate source of energy [25-28]. This also explains the increase in corn intake in camels group fed Atriplex.

Our results indicated that camels fed on Atriplex and hay had higher values of nitrogen intake and digested nitrogen more than straw. These could mainly be due to the type of forage and its content of [29,23]

The roughage type significantly affected the water intake and excretion. Atriplex camels group recorded the highest free water intake. Because Atriplex had high salt content [30]. Camels can be fed on a high tolerance saline fodder than any other animal [31]. Camels fed this diet drink a lot of water in order to eliminate the salts ingested. 
Atriplex camels group recorded significantly higher feed water intake than those hay and straw groups due to that Atriplex had higher moisture content about $65 \%$ similar findings were recorded by [21]. Camels consumed Atriplex recorded higher water execration values than those fed on straw or hay. These results indicated that camels' kidneys seem to be better adapted to handling salt load especially when they fed on halophytic plants $[32,33]$ pointed out that increasing water excretion through the urinary pathway is believed to be an adaptive mechanism assisting the animal in getting rid of excess salts and maintain osmolality of food and other body fluids. In addition, [34,35] reported that increasing salts increased water excretion in urine and faeces.

\section{CONCLUSION}

In conclusion, the results indicate that drinking saline water did not affect feed intake, digestibility and nitrogen utilization. whereas, roughages type had effect intake, digestibility and nitrogen utilization. Camels fed on Atriplex showed a clear improvement in growth, digestibility, and nitrogen utilization in a similar way to camels fed on hay. As for the camels fed on straw, they had the least effect on growth, digestibility, and nitrogen utilization.

\section{REFERENCES}

[1] Al-Dahash S, Sassi M (2009). A preliminary study on management, breeding and reproductive performance of Camel in Libya. Iraq J Vet Sci 23: 276-281.

[2] Kadim, I. T., Mahgoub, O., Al-marzooqi, W., Khalaf, S. K., and Raiymbek, G. (2013). Composition, quality and health aspects of the dromedary (Camelus dromedarius) and bactrian (Camelus bacterianus) camel meats: a review. J. Agric. Mar. Sci. 18, 7-24.

[3] Faye, B. (2015). Role, distribution and perspective of camel breeding in the third millennium economies. Emirates J. Food Agric. 27, 318-327.

[4] Ghali, M.B., Scott, P.T., Alhadrami, G.A. and Al Jassim, R.A.M., 2011. Identification and characterisation of the predominant lactic acidproducing and lactic acid-utilising bacteria in the foregut of the feral camel (Camelus dromedarius) in Australia. Animal Production Science, 51(7), pp.597604.

[5] Rabee, A.E., Forster, R.J., Elekwachi, C.O., Kewan, K.Z., Sabra, E.A., Shawket, S.M., Mahrous, H.A. and Khamiss, O.A., 2019. Community structure and fibrolytic activities of anaerobic rumen fungi in dromedary camels. Journal of basic microbiology, 59(1), pp.101-110.

[6] Kayouli, C., Jouany, J.P., Demeyer, D.I., Taoueb, H. and Dardillat, C., 1993. Comparative studies on the degradation and mean retention time of solid and liquid phases in the forestomachs of dromedaries and sheep fed on low-quality roughages from Tunisia. Animal feed science and technology, 40(4), pp.343-355.

[7] Khattab, I.M., Abdel-Wahed, A.M., Anele, U.Y., Sallam, S.M. and El-Zaiat, H.M., 2021. Comparative digestibility and rumen fermentation of camels and sheep fed different forage sources. Animal Biotechnology, pp.1-10.

[8] Payne, W.J.A., 1990. An introduction to animal husbandry in the tropics (No. Edn 4). Longman scientific and technical.

[9] Gauthier-Pilters, H. and Dagg, A.I., 1981. The camel. Its evolution, ecology, behavior, and relationship to man. The University of Chicago Press.

[10] Gaughan JB (2011) Which physiological adaptation allows camels to tolerate high heat load and what more can we learn? J Camelid Sci 4: 85-88.

[11] MacFarlane, W.V., Morris, R.J.H., Howard, B., 1963. Turnover and distribution of water in desert camels, sheep, cattle and kangaroos. Nature 197, 270-271.

[12] Schmidt-Nielsen, K., 1964. Desert Animals Physiological Problems of Heat and Water. Oxford University Press, Oxford, pp. 33-70.

[13] Wilson, R.T., 1989. Ecophysiology of the Camelidae and Desert Ruminants. Springer Verlag, Berlin.

[14] Robertshaw, D., Zine-Filali, R., 1995. Thermoregulation and water balance in the camel: a comparison with other ruminant species. In: von Engelhardt, W., Leonhard-Marek, S., Breves, G., Giesecke, D. (Eds.), Ruminant Physiology: Digestion, Metabolism, Growth and Reproduction. Ferdinand Enke Verlag, Stuttgart, pp. 565-778.

[15] Farid, M.F.A., A.M. Abdel-Wahed, Safinaz M. Shawket and N.I, Hassan. (2010). Diet Selection, feed Intake capacity and performance of growing female camels: Effects of type of roughage and level of concentrates offered. J. American Sci., 6: 317-326

[16] AOAC - Association of Official Agricultural Chemists. (1990). Official Methods of Analysis, 15th edition. AOAC, Washington DC

[17] Hintze, (2007). NCSS. Kaysville, Utah, U.S.A.

[18] Abdel-Wahed, A. M. (2014). Effect of type of roughage of concentrate supplements on feed digestion and utilization in growing female dromedary camels. Journal of American Science 2014;10 (6): 198-206.

[19] Cianci, D., L. Goio, A.M. Hashi, S. Pastorelli, M. Kamoun, G.B. Liponi and M. Orlandi. (2004). Feed intake and digestibility in camels fed wheat straw and Meadow hay. J. Camel Science. 1: 52-56.

[20] Assad, F., El-Sherif, M.M.A., 2002. Effect of drinking saline water and feed shortage on adaptive responses of sheep and camels. Small Ruminant Research, 45, 279290

[21] Abdel-Wahed, A.M., 2020. Water intake and excretion of growing she-camels in relation to the type of roughage fed and concentrate. International Journal of Environment, Agriculture and Biotechnology, 5,(4) Pages 953-964 
[22] Shawket, S. M., K.M. Youssef and M. H. Ahmed. (2010). Comparative evaluation of Egyptian clover and Atriplex halimus diets for growth and milk production in camel. Animal Science Reporter, vol. 4: 9-21.

[23] Shawket, S.M., Khattab, I.M., Borhami, B.E. and ElShazly, K.A., 2001. Performance of growing goats fed halophytic pasture with different energy sources. Egyptian J. Nutrition and feeds, 4, 251-264.

[24] Chamberlain, A. (1989). Milk production in the tropics. Intermediate Tropical Agricultural Series 13, Camels, 202-210

[25] Cherdthong, A., Wanapat, M. and Wachirapakorn, C., 2011. Influence of urea-calcium mixtures as rumen slow-release feed on in vitro fermentation using a gas production technique. Archives of animal nutrition, 65(3), pp.242-254.

[26] Khattab, I.M., Salem, A.Z.M., Abdel-Wahed, A.M. and Kewan, K.Z., 2013. Effects of urea supplementation on nutrient digestibility, nitrogen utilisation and rumen fermentation in sheep fed diets containing dates. Livestock Science, 155(2-3), pp.223-229.

[27] El-Zaiat, H.M., Kholif, A.E., Khattab, I.M. and Sallam, S.M., 2021. Slow-release urea partially replace soybean in the diet of Holstein dairy cows: intake, blood parameters, nutrients digestibility, energy utilization, and milk production. Annals of Animal Science.

[28] Khattab, I.M. and Anele, U.Y., 2021. Dry matter intake, digestibility, nitrogen utilization and fermentation characteristics of sheep fed Atriplex hay-based diet supplemented with discarded dates as a replacement for barley grain. Journal of Animal Physiology and Animal Nutrition

[29] EI-Shaer, H.M. and H.M. Kandil. (1990). Comparative study on the nutritional value of wild and cultivated Atriplex halimus by sheep and goats in Sinai. Com. Sci. and Dev. Res., 29: 81-90.

[30] Khattab, I.M., 2007. Studies on halophytic forages as sheep fodder under arid and semi arid conditions in Egypt, Ph. D. Thesis. Alexandria University.

[31] Squires, V.R., 2015. Halophyte and Salt Tolerant Plants Feeding Potential to Dromedary Camels. In Halophytic and Salt-Tolerant Feedstuffs (pp. 273-284). CRC Press.

[32] Abou El-Nasr, H.M., M. Shawket, Safinaz, M.F. Farid and H.S. Khamis. (1988). Studies on saline drinking water for camels and sheep: 1- Water intake and excretion. Alex. J. Com. Sci. Dev. Res., 23: 131-139

[33] Ahmed, M.H. (1984). Studies on nitrogen metabolism in ruminants: effects of drinking saline water on nitrogen metabolism and rumen kidney function in sheep. Ph. D. Thesis, Alex. Univ., Egypt.

[34] El-Faramawi, A.A. (1984). Effect of drinking bring water on energy and water metabolism in sheep. M. Sc. Thesis, Ain Shams Univ

[35] Kandil, H.M., A.O. Sooud, M.FA. Farid, H.M. ElShaer, and M.A. El-Ashry. (1985). Effect of drinking saline water on feed utilization and nitrogen and mineral balance in camels. First Inter. Congress on
Animal Production in Arid zones 9 ZICAPAZ, Sep., 7 14, 1985, ACSAD, Damascus, Syria. 